\title{
A Survey of Statistical Source Models for Variable Bit-Rate Compressed Video
}

\author{
Michael R. Izquierdo ${ }^{\dagger}$ and Douglas S. Reeves ${ }^{\ddagger}$ \\ ${ }^{\dagger}$ IBM Corporation, Research Triangle Park, NC 27709 \\ ${ }^{\ddagger}$ North Carolina State University, Raleigh, NC 27695
}

\begin{abstract}
It is predicted that in the near future, the transport of compressed video will pervade computer networks. Variable bit-rate (VBR) encoded video is expected to become a significant source of network traffic, due to its advantages in statistical multiplexing gain and consistent video quality. Both systems analysts and developers need to assess and study the impact these sources will have on their networks and networking products. To this end, suitable statistical source models are required to analyze performance metrics such as packet loss, delay and jitter.

This paper provides a survey of VBR source models which can be used to drive network simulations. The models are categorized into four groups: Markov Chain and Autoregressive, TES, Self-Similar and i.i.d/Analytical. We present models which have been used for VBR sources containing moderate to significant scene changes and moderate to full motion. A description of each model is given along with corresponding advantages and shortcomings. Comparisons are made based on the complexity of each model.
\end{abstract}

Keywords: Video Modeling, VBR, Variable Bit Rate, MPEG, H.261, TES, Self-Similar.

This work was supported by the Air Force Office of Scientific Research under Grants F49620-92-J-0441 and F49620-96-1-0061, and by an IBM Corporation Resident Study Fellowship. To contact the authors please send email to mrizquie@ raleigh.ibm.com or reeves@eos.ncsu.edu 


\section{Introduction}

The recent development of standards for digital video compression, such as H.261 [3], H.263, [21] MPEG-1 [19] and MPEG-2 [20], has made it feasible to transport video data over computer communications networks. It is predicted that in the near future, transporting video over computer networks will become commonplace. Classical models based on a Poisson arrival process, traditionally used in the analysis of telephony networks, are not adequate to model video traffic. This is due to the fact that the Poisson process assumes that arrivals are independent, whereas for compressed video they are not. In consequence, new models are needed to describe compressed video sources, and derive attendant performance measures.

Raw compressed video is a traffic source which can have high peak-to-mean ratios and significantly high autocorrelations. This type of source can be deleterious to networks, since it can cause severe data loss if network resources are not properly allocated. One way to ameliorate this difficulty is to control the output bit-rate of the encoder. This is referred to as Constant bit-rate (CBR) encoding. A CBR encoder's output bit-rate is nearly constant, making it possible to transport its output using a fixed-rate channel. This makes bandwidth allocation simpler and also renders the video source more amenable to traffic policing. However, CBR encoding has the drawback that video quality (distortion) varies significantly in order to maintain a constant bit-rate. Also, the desired bit-rate needs to be determined up-front which for some applications, such as the real-time encoding of a live sporting event, might not be optimal since a worst case bit-rate might be chosen.

An alternative to CBR encoding is Variable Bit Rate (VBR) encoding. VBR does not attempt to control the output bit-rate of the encoder, so distortion ${ }^{1}$ does not vary significantly. One way to accomplish this is to keep the quantizer step size fixed. However, this makes the output bit-rate of VBR encoders vary considerably, making bandwidth alloca-

1. Distortion is a coarse measure of video quality and is measured in terms of signal-to-noise ratio or SNR. 


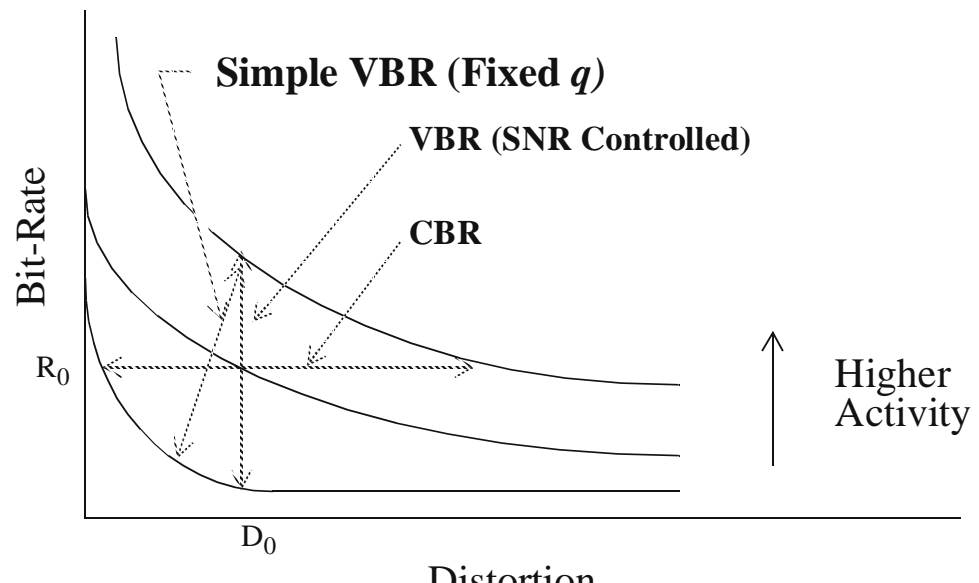

Distortion

Figure 1. Distortion versus bit-rate curves for compressed video.

tion difficult. On the other hand, this variability increases the opportunity for improvements in statistical multiplexing gain $\left(\mathrm{SMG}^{2}\right)$. Figure 1 depicts the basic trade-off between distortion versus bit-rate for compressed video. We observe that distortion varies as an encoder controls the bit-rate (CBR), but if the encoder controls distortion (VBR), then bitrate varies. This behavior highlights the basic differences between CBR and VBR encoding.

Increases in SMG occurs due to the fact that the likelihood of multiple bursty sources simultaneously transmitting at their peak bit-rates is small. One of the first investigations regarding the SMG of encoded video was done by Haskell, who found that a 2:1 gain was achievable when multiplexing the outputs of several AT\&T Picturephone ${ }^{\circledR}$ encoders [10]. The SMG of VBR sources has been further quantified in early works by Kishino [24], Morrison [38] and Verbiest [45], where gains of up to 4:1 were obtained for simple sequences.

2. In the literature, definitions of SMG has arisen. One is the ratio of the multiplexer output link utilization for N VBR sources compared to only one source, given the same packet (cell) loss probability. The second defines SMG as the ratio of the number of VBR sources to the number of CBR sources (preferably encoded using the same source material; however, this is often not the case and is usually estimated) which a multiplexer can accommodate given the same link capacity. 
One drawback to multiplexing VBR sources is the increased possibility of packet loss. Such losses result when multiple independent VBR sources cause the multiplexing buffer to overflow. Analyzing the multiplexer buffer occupancy behavior is a major concern for researchers. Simulation studies are often used in order to quantify the amount of loss, since analytic methods are often intractable. In order to run these simulations, source models are used to provide input stimulus to the system under study. While actual video traces may be used in place of a source model, this limits the input to a finite realization of the underlying stochastic process, which reduces the generality of the simulation results.

In this paper, we survey statistical source models for VBR video which have been proposed for both video conferencing and movie sequences. We define video conference models as those being encoded using either H.261 or MPEG without $B$ frames. A movie sequence is one which is encoded using MPEG with I, B and P frames. Some examples of models presented for movie sequences are: Star Wars, ${ }^{3}$ Last Action Hero, ${ }^{4}$ and The Wizard of $\mathrm{Oz}^{5}$ Only MPEG-1 models were covered in this survey since a viable MPEG-2 model had not been published by the time this survey was concluded. For the most part, the models covered in this survey should apply to MPEG-2 equally as well. For introductory material on the encoding standards H.261 and MPEG-1, refer to [31] and [28], respectively.

The motivation underlying the choice of these particular models is to present a representative sampling of current VBR source models. The models are grouped into four categories (AR/Markov, TES, Self-Similar, Analytical/i.i.d.) and then presented in chronological order. This was done so that the evolution of VBR modeling was readily discernible. We provide detailed descriptions of each model, where necessary, and discuss the motivations and merits of each model. We also describe how each model was validated and the number of parameters used by the model.

3. Lucasfilm Ltd./20th Century Fox, 1977.

4. Columbia Pictures, 1993.

5. MGM, 1939. 
The paper is organized as follows. Section 2 discusses on statistical modeling and model validation. Section 3 covers models based on Markov Chains and Autoregressive processes (AR). Section 4 covers models based on the TES process. Section 5 describes models based on self-similar processes. Section 6 presents analytical and non-Markovian models which are based on i.i.d. processes. Section 7 summarizes the paper, including a comparison of the number of parameters required by each model in this survey. Recommendations and issues relating to model selection are also discussed, as well as suggestions for further research in VBR modeling. 


\section{Statistical Modeling and Model Validation}

A good model is one which can accurately predict performance measures (statistics) of a stochastic system. For example, if the researcher is interested in the cell loss probability for an ATM buffer with VBR sources, then a good source model is one which produces a sample path which can accurately predict this performance measure, when the system is simulated. In most cases the validity or "goodness" of a model is determined by comparing model predictions (e.g. simulation statistics using the empirical data as the traffic source) and the corresponding statistics using the model as the traffic source. It is possible for a model to predict one metric accurately and another inaccurately. For example, a model may provide accurate predictions for cell loss probability, and be inaccurate predicting mean cell delay. The researcher must decide beforehand what the desired system metrics should be, and select a model which can accurately predict these metrics.

There are reasons to argue that the validation of a source model requires that its distribution and autocorrelation function match well their empirical counterparts, while using as few parameters as possible ${ }^{6}$. Typically, one tries to fit the empirical data with a classical distribution such as Log-Normal or Gamma, but whether or not a good fit is found, the empirical distribution (say, histogram) is a good fit by default. A common method used to match distributions is the QQ plot. Matching the autocorrelation function is a more difficult task. The autocorrelation function is a proxy for the temporal (linear) dependence within a stochastic process. Generally, stochastic processes may be classified into three types: independent, short-range dependent (SRD) and long-range dependent (LRD). An independent source is always uncorrelated, i.e., is identically zero for positive lags; the converse is false, namely, lack of correlation does not imply independence. If the autocorrelation function is summable (e.g. when it decays exponentially fast), then it is referred to as an SRD process, but if it is not summable (e.g., when it decays hyperbolically), then the

6. Exact matching (at all lags) of the autocorrelation function is a subject of on-going debate (see Heyman [11]) 
source is referred to as an LRD process. The requirement to use few parameters is motivated by the fact that they must be estimated from the empirical data. Each estimate incurs a certain amount of error which tends to reduce the accuracy of the model as the number of parameters increases (although those errors may occasionally cancel out).

In this survey, we classify the models into two types: hierarchical and non-hierarchical. Models which capture scene changes explicitly are referred to as hierarchical models. A scene change process aims to model the relative frequency of individual scene types over longer time scale (minutes or hours) than the bit-rate process (milliseconds). Scene changes occur when the mean of the bit-rate process changes significantly as a result of a considerable change in picture content (camera cuts).

The parsimony of a model is determined by the number of parameters it requires and its complexity by the amount of computer time and memory required to generate a sample. Models which require many parameters generally require many calculations in order to generate a sample, but there are exceptions (e.g., TES). On the other hand, some models require few parameters, but take a long time to generate each sample, since each sample is calculated from all previous samples (e.g. LRD and self-similar models). It is desirable to develop a model of minimal complexity which provides sufficiently accurate predictions of the metrics of interest. 


\section{Models Based on Markov Chains and Autoregressive Processes}

We provide a brief review of Markov Chains and Autoregressive (AR) processes since many of the VBR source models are based on them. Both processes incorporate temporal dependence. AR processes, in most case, use Gaussian random variables, producing sequences which are Normally distributed. Markov Chains, those achieving steady-state, can produce a wide variety of distributions.

\subsection{Review of Markov process}

Models based on a Markov process use states to represent bit-rate regimes (roughly a range of bit-rates of a video sequence). A stochastic process $\left\{X_{k}\right\}, k=1,2, \ldots$ with state space $S=\{1,2,3, \ldots\}$ is Markovian if for every $n$ and all states $i_{1}, i_{2}, \ldots$, where $i_{n} \in S$ it satisfies the Markov property,

$$
P\left[X_{n} \mid X_{n-1}=i_{n-1}, X_{n-2}=i_{n-2}, \ldots, X_{1}=i_{1}\right]=P\left[X_{n}=i_{n} \mid X_{n-1}=i_{n-1}\right]
$$

Simply put, the current state of a Markov process depends only on its previous state and not on any additional previous states. A stochastic process is called a Markov Chain if the state space is countably infinite or finite.

As an example, a continuous-time discrete-state Markov process behaves as follows: it enters a state and remains there for an exponential period of time whose parameter $\lambda_{i}$ depends on the state $i$. At the end of this period, the process moves to a different state governed by the Markov property. Transitions between states are controlled by a transition probability matrix; corresponding transition probabilities are estimated from the actual video trace. Steady-state distributions, if any, are determined from the transition matrix. For a more complete discussion of Markov Chains, see [18]. 
Markov Chains are often used to modulate other processes such as Bernoulli, Poisson or AR. The state of the Markov Chain represents a different set of parameters for the particular process. While in a particular state, the model generates samples according to the particular process (Bernoulli, etc.), at the specific parameter settings. This is done for a period of time until the process switches to a different state, generating samples using a different set of parameters. Models of this type are referred to as Markov modulated or Markov modified models. Some examples of such models are the Markov Modulated Bernoulli Process (MMBP) and the Markov Modulated Poisson Process (MMPP). We will show many examples of video models which use Markov modulation.

\subsection{Review of autoregressive process}

In an AR process, the current value is a function of a weighted linear combination of past values. Formally, it is expressed as

$$
x(n)=a_{0}+\sum_{i=1}^{p} a_{i} x(n-i)+e(n),
$$

where $a_{0}$ is called the intercept and $\left\{a_{1}, a_{2}, \ldots, a_{p}\right\}$ are AR coefficients, $p$ is the order of the $\mathrm{AR}$ process and $e(n)$ are the residuals, commonly assumed uncorrelated and Normally distributed. The AR coefficients can be determined using the recursive Levinson-Durbin algorithm [40, Appendix 2A]. AR processes of order $p$ are denoted by $A R(p)$. A special case of (2) is the $\mathrm{AR}(1)$ process

$$
x(n)=a_{0}+a_{1} x(n-1)+e(n),
$$

where $a_{1}$ is the autocorrelation coefficient at lag-1 when the sequence is stationary. Note that (3) can be seen as a continuous-state, discrete-time Markov process. A model of this form was presented in [32]. 
The AR process is a special case of the autoregressive moving average process (ARMA) which adds a moving average process (MA) giving

$$
x(n)=a_{0}+\sum_{i=1}^{p} a_{i} x(n-i)+\sum_{j=0}^{q} b_{j} e(n),
$$

The ARMA process is typically stated as ARMA(p,q) where $p$ is the order of the AR part and $q$ is the order of the MA part. Determining the coefficients, $b_{j}$, is a bit more involved than an AR process and usually requires some form of spectral analysis. [9]

\subsection{ATM cell-level model using ARMA}

Grunenfelder, et al. [9] developed a model, from a four second video conference sequence, for the ATM cell interarrival process from a video encoder using conditional replenishment ${ }^{7}$. The model defined a fixed time interval of 64 slots, where 1 slot equaled the time to transmit a 36 byte cell. The random process, $\left\{X_{i}\right\}$, defined the number of cells generated by the encoder within this interval, where

$$
\begin{aligned}
X_{i}=g\left(\alpha Z_{i-m}+Y_{i}+v_{i}\right) \quad|\alpha|<1 \\
Y_{i}=\sum_{k=-m / 2}^{m / 2} h_{k} \varepsilon_{i-k}
\end{aligned}
$$

where $v_{i}$ is white noise. The parameters for the model were estimated from the long-term mean, variance and autocovariance of the empirical sequence. The coefficients, $h_{k}$, of the MA part were determined using Fourier analysis. The ARMA process was referred to as a colored-Gaussian process with zero mean, unit variance which implies that the autocorrelation function is not from a pure Gaussian process.

7. Encoders using conditional replenishment only transmit the difference in the pixel areas between a reference and current frame. This is done using differential pulse code modulation (DPCM). Areas which do not change are run length coded (RLC). When this difference becomes excessive, a new reference frame is generated and transmitted. 
In digital signal processing terms, the ARMA sequence is generated using the ARMA filter with white noise as input. Since the original sequence is not a zero mean process, the output of the filter, $\left\{V_{i}\right\}$, is transformed using a zero-mean nonlinearity (ZMNL) function, $\mathrm{g}($.$) , of the form a V_{i}+b$.

This model requires 10,003 parameters, $\left(\alpha, a, b, h_{1}, h_{2}, \ldots h_{10,000}\right)$, where the MA coefficients cover approximately seven frames. Model parameters were estimated from four seconds of video. This model can be viewed as modeling the video sequence at the sub-frame layer (slice/GOB) and it matches the pseudo-periodic autocorrelation function, typical of these sequences, quite well.

\subsection{Video conference model using Markov Chain}

Heyman, et al. [13] developed a frame level ${ }^{8}$ ATM model of a 30 minute video conference sequence with no scene changes and moderate motion. The model defines the number of ATM cells per frame, $X_{n}$, and the state of the Markov Chain, $Y_{n}$, where $Y_{n}=\left\lfloor X_{n} / 10\right\rfloor^{9}$. The transition probability matrix, $P=\left[p_{i j}\right]$, was estimated using,

$$
p_{i j}=\frac{\text { number of transitions from state } \mathrm{i} \text { to state } \mathrm{j}}{\text { number of transitions out of state } \mathrm{i}} .
$$

This model requires many parameters due to the transition probability matrix. In order to reduce the number of parameters, the authors used the Discrete Autoregressive Process (DAR) which estimates the transition probabilities by using the empirical marginal distribution and autocorrelation coefficient. The transition matrix is given by

$$
P=\rho I+(1-\rho) Q
$$

8. The frame level corresponds to MPEG or H.261 pictures.

9. $\lfloor x\rfloor$ is the floor function, where $x$ is rounded towards $-\infty$ 
where $\rho$ is the autocorrelation at lag $1, I$ is the identity matrix, and each row of $Q$ consists of the marginal probability distribution function (pdf) of the empirical data. Since the empirical data was found to fit a negative-binomial distribution, each row in $Q$ contained the probabilities $\left(f_{0}, f_{1}, \ldots, f_{K}, f_{K}^{c}\right)$ defined by,

$$
\begin{gathered}
f_{k}=\left(\begin{array}{c}
k+r+1 \\
k
\end{array}\right) p^{r}(1-p)^{k} \\
f_{K}^{c}=\sum_{k>K} f_{k}
\end{gathered}
$$

where the parameters $r$ and $p$ are estimated from the empirical data, and $K$ is the maximum number of cells in a frame. By using DAR the number of parameters was reduced to only four: peak, mean, variance, and autocorrelation at lag 1.

Interestingly, this model gave rise to better bit-rate predictions than a second order AR process which was also proposed at the time. This model is suitable for video conferences with no significant scene changes, since it does not model the scene changes explicitly. The model does rely on the use of a classical distribution (negative-binomial) in DAR; however, the empirical distribution could be used.

Lucantoni, et al. [27] proposed a model using a discrete-state, continuous-time Markov Renewal Process (MRP), which they compared to the DAR model. This model is of the same vein as MMPP, but instead the bit-rate is fixed, not probabilistic. They divided the range of possible rates into 40 equidistant levels and assigned a state in the Markov Chain to each level. One, and sometimes two, geometric distribution were fitted to sojourn time at each level. Sample paths generated by the model are strikingly similar in appearance to the empirical trace data. They compared the leaky bucket contour curves, generated using both models, and found that MRP was better than DAR in approximating the results produced when using the empirical sequence. 


\subsection{Hierarchical model using composite AR processes and Markov Chain}

Ramamurthy and Sengupta [41] proposed a hierarchical model which uses a Markov Chain to capture the effects of a scene change. The model consists of two AR processes where the first attempts to match the autocorrelation function at short lags and the second, the autocorrelation at long lags. The third process is a Markov process which is used for scene changes. Combining the three processes gives the final model

$$
T_{i}=X_{i}+Y_{i}+Z_{i}
$$

where

$$
\begin{gathered}
X_{i}=a_{1} X_{i-1}+A_{i}, \\
Y_{i}=a_{2} Y_{i-1}+B_{i} \text { and } \\
Z_{i}=K_{i} C_{i}
\end{gathered}
$$

Equation (10) is used to generate a sequence whose autocorrelation function matches that of the emprical sequence at short lags while (11) matches it at long lags. Bothh equations are $\mathrm{AR}(1)$ processes, where $A_{i}$ and $B_{i}$ are normally distributed with means: $\mu_{1}, \mu_{2}$ and standard deviations: $\sigma_{1}, \sigma_{2}$. Equation (12) is used to generate the extra bits needed when a scene change occurs, where $K_{i}$ represents the state of the Markov Chain and $C_{i}$ is a normally distributed random variable whose mean and variance depends on $K_{i}$. The number of parameters required for $C_{i}$ is reduced from four to two by making the mean and variance a function of $\alpha$ and $\beta$, where $\mu=\alpha, \sigma=\beta$ when $K_{i}=2$ and $\mu=\alpha / 2, \sigma=\beta / 2$ when $K_{i}=1$. The basic premise behind the use of the Markov Chain, shown in Figure 2, is to generate the extra bits needed in the two frames following a scene change. We will see later a model proposed by Heyman and Lakshman which also takes into consideration the 
first two frames after a scene change. This model requires a total of eight parameters $\left(a_{1}, \mu_{1}, \sigma_{1}, a_{2}, \mu_{2}, \sigma_{2}, \alpha, \beta\right)$

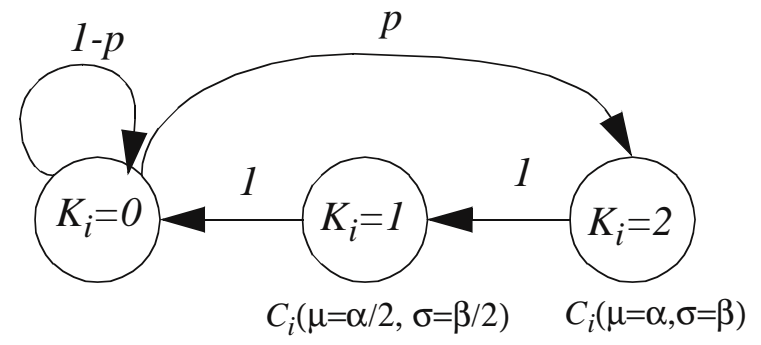

Figure 2. Markov Chain of scene change process.

\subsection{MPEG frame and slice layer models using Markov Chains}

Pancha and El Zarki [39] proposed an MPEG frame and slice layer Markov Chain model for a 3 minute, 40 second sequence of the movie Star Wars. This model differs from Heyman's model in that rather than each state representing the number of cells in a frame, each state represents a bit-rate change of one standard deviation. This is illustrated in Figure 3. Transition matrices were given for different GOP sizes and, in general, the larger the GOP size the more states required in the Markov Chain. Also, the slice layer required more states than the frame layer. The number of parameters required by the models ranged from 51 for the frame layer to 102 for the slice layer, where the mean and standard deviation was estimated from the empirical trace data and added to the number of transition probabilities which we summarize in Table 1.

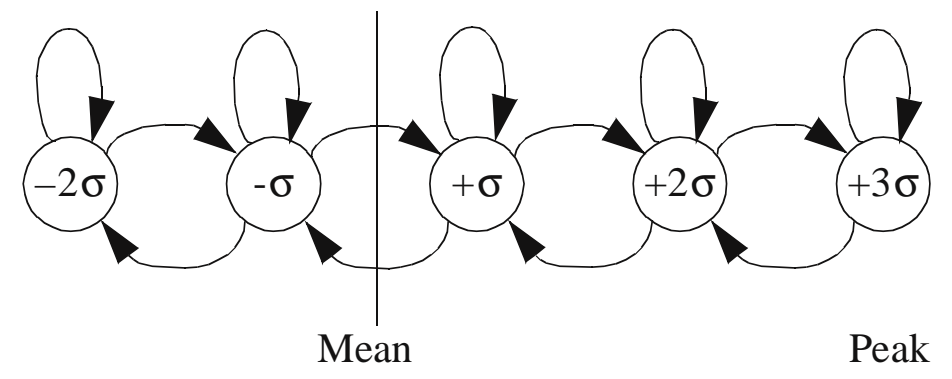

Figure 3. Markov chain model for video sources. 
Table 1. Transition probability count for frame and slice layer models.

\begin{tabular}{|c|c|c|c|}
\hline \multirow{2}{*}{ GOP Size (N) } & Frame Type & \multicolumn{2}{|c|}{ Transition Probabilities } \\
\cline { 3 - 4 } & Frame & Slice \\
\hline 1 & I & 49 & 64 \\
\hline 16 & I,P & 64 & 81 \\
\hline 2688 & I & 64 & 100 \\
\hline
\end{tabular}

\subsection{Video conference model using composite AR processes}

Yegenoglu, et al. [46] analyzed a full-motion color video sequence of 500 frames encoded by discrete cosine transform (DCT), differential pulse code modulation (DPCM) and motion compensation. The picture resolution was $720 \times 480$ pixels with 16 bits per pixel and the rate was 30 frames per second. The primary motivation of this model was to produce a multi-modal probability density function (in this case, Gaussian). Previous work indicated that the probability density functions of VBR video conference streams appeared to consist of a combination of probability distribution functions; indeed the model did produce a multimodal probability density function.

The model is based on an AR process whose parameters are modulated by a Markov Chain. The model quantizes bit-rates into $N$ levels where a quantization level loosely corresponds to a scene class. The Markov Chain defines the transition process between quantization levels, where a single distinct $\mathrm{AR}(1)$ process is defined for each state of the Markov Chain. When the bit-rate crosses a quantization level, the first frame of the new quantization level is sampled from an i.i.d. Gaussian random variable.

The state of the Markov Chain at a particular time instant, $t$, is defined as $x_{t}$, where $x_{t} \neq x_{t-1}$ signifies a state change has occurred. For each state, $i$, there are a unique set of 
coefficients which is used to determine the number of bits per frame, $y_{t}$, given by the regressive relation,

$$
y_{t}=\left\{\begin{array}{cl}
a(i) y_{t-1}+G\left(\mu(i), \sigma(i)^{2}\right), & \text { if } x_{t}=x_{t-1}=i \\
G(\eta(i), v(i)), & \text { if } x_{t} \neq x_{t-1} ; \mathrm{x}_{t}=i
\end{array}\right.
$$

where $G($.$) is a Gaussian random variable and a(i)$ is the autocorrelation coefficient at lag one at state $i$. When a state transition occurs, a sample is drawn from $G(\eta(i), v(i))$, where the mean and variance of the bit-rate process is conditioned on the state of the Markov Chain, $i$. The parameters for (13) are calculated using the following constraints

$$
\begin{gathered}
a(i)=1-\frac{D^{2}(i)}{2 v(i)}, \\
\mu(i)=\frac{\eta(i)\left(D^{2}(i)\right)}{2 v(i)}, \\
\sigma(i)^{2}=D^{2}(i)\left(1-\frac{D^{2}(i)}{4 v(i)}\right),
\end{gathered}
$$

where

$$
D^{2}(i)=E\left[\left(y_{t}-y_{t-1}\right)^{2} \mid x_{t}=x_{t-1}=i\right]
$$

is the conditioned expected square difference of the bit-rates in adjacent frames. This parameter allows for the empirical data to be characterized in one pass, rather than the two passes it would have otherwise required. The values $\eta(i), v(i)$ and $D^{2}(i)$ are estimated from the empirical data. 
The probability density function, $f_{Y}(y)$, of the number of bits per frame, $y_{t}$, is approximated by a combination of $N$ Gaussian densities

$$
f_{Y}(y)=\sum_{i=1}^{N} p_{i} f_{G(\eta(i), v(i))}(y)
$$

where $p_{i}$ is the steady-state probability of state $i$ in the underlying Markov Chain.

Three states were used to represent the quantizations $(0,44),(44,55)$ and $(55, \infty) \mathrm{kbits} /$ sec, resulting in good agreement between the distributions of the model and empirical data using the Kolmogorov-Smirnov test. The model captured the first, second and fourth moments of the actual data; however, the third moment in one particular state differed significantly. This was due to the fact that the actual data was not symmetrical about the mean beyond $55 \mathrm{kbits} / \mathrm{sec}$.

This model requires a total of nine parameters, three for each quantization level $\left\{D^{2}(i), \eta(i), v(i)\right\}$ where $i=\{1,2,3\}$. It is useful for video conference sequences with small to moderate motion and scene changes. The tricky part is to determine the appropriate quantization levels, a task that requires visual inspection of the empirical bit-rate distribution.

\subsection{Block-based video conference model using AR processes}

Jabbari et al. [22] developed a block-based bit-rate model of a video encoder which adhered to the general MPEG syntax. The encoder differs from the recommended MPEG implementation in that the resolution is CCIR (720x480 pixels/frame) instead of SIF (360x240 pixels/field). Interlaced scanning is used, producing two fields for every frame. In most aspects, the encoder is more similar to MPEG-2 than to MPEG-1. The statistics of encoded blocks contained within $I$ and $P$ frames were estimated for a sequence of 350 fields (175 frames) (the model does not account for B frames). The model divides each field into three block types: 0,1 and 2 where a block is defined as an $8 \times 8$ matrix of pixels. 
Type 0 blocks are encoded using DPCM and are used for sequences with very little motion. Type 1 blocks use motion compensation on sequences containing moderate motion. Type 2 blocks use DCT on sequences with high motion. I frames contain only type 2 blocks while, $P$ frames contain all three types.

The model consists of a vector-valued sequence

$$
\begin{aligned}
& x_{0}(n)=a_{0} x_{0}(n-1)+e_{0}(n), \\
& x_{1}(n)=a_{1} x_{1}(n-1)+b_{0} x_{0}(n)+b_{2} x_{2}(n)+e_{1}(n), \\
& x_{2}(n)=a_{2} x_{2}(n-1)+e_{2}(n),
\end{aligned}
$$

whose components represent the number of Type 0,1 and 2 blocks in each frame, $n$ is the frame index and $e_{i}(n)$ is a Gaussian random variable with mean $\mu_{i}$ and variance $\sigma_{i}^{2}$ for $i=0,1$ and 2 . The determination of the parameters for (19) is involved and is not included here; but details can be found in [22].

The average number of bits for block type $i$, within frame $n$, is given by

$$
r_{i}(n)=c_{i} r_{i}(n-1)+g_{i}(n),
$$

where $c_{i}$ is the first order AR coefficient and $g_{i}$ is a Gaussian random variable. This is used to find the total bits per block type $i$ within frame $n$ given as

$$
u_{i}(n)=r_{i}(n) x_{i}(n) . \quad i=0,1 \text { and } 2,
$$

The final model for the total number of bits per frame, $u_{T}(n)$, is therefore,

$$
u_{T}(n)=u_{0}(n)+u_{1}(n)+u_{2}(n) .
$$

The model produced sample data which was shown to match the distribution of the actual data well via a QQ-plot. However, the model was not used in the simulation of a multiplexer and the autocorrelation function of $u_{T}$ was not given. A total of 20 parameters are required for this model, of which 


$$
\left\{a_{0} \mu_{0}, \sigma_{0}, a_{1}, b_{0}, b_{2}, \mu_{1}, \sigma_{1}, a_{2}, \mu_{2}, \sigma_{2}\right\}
$$

are used for the number blocks per frame, and $\left(c_{i}, \mu_{i}, \sigma_{i}\right), i=\{0,1,2\}$, are used for the number of bits per block.

\subsection{Hierarchical model using the DAR process}

Heyman and Lakshman [12] proposed an ATM frame layer model for sequences generated using a DPCM codec (motion compensation was not used) consisting of three different stochastic processes: (1) Scene length, (2) Size of the first frame after a scene change, and (3) Size of frames within a scene. Scene change boundaries were determined using the second difference

$$
\Delta_{i}=\frac{\left(X_{i+1}-X_{i}\right)-\left(X_{i}-X_{i-1}\right)}{\frac{1}{m} \sum_{k=1}^{m} X_{i-k}}
$$

where $X_{i}$ is the number of bits in frame $i$. A scene change is defined to occur when $\Delta_{i}$ is large and negative ( $m$ was heuristically determined). The scene change process was determined to be uncorrelated; consequently, matching the distribution was sufficient. The first frame after a scene change was found to follow a different stochastic process since the scene content completely changes causing the frame to be regenerated. It was found that scene length distributions fit Weibull, Gamma and Pareto distributions well while the number of cells within a scene change frame fit Weibull, Gamma, and in one case Normal distributions (some sequences, however, did not appear to fit any distribution). The frame following a scene change frame was generated using linear prediction of the form

$$
Y_{i}=a+b X_{i}+\varepsilon_{i}
$$

where $a$ and $b$ are fixed coefficients and $\varepsilon_{\imath}$ is white noise. 
Since the frame size within a scene is correlated, a Markov Chain was used where each state represented the integer part of $X_{i} / 50$ and the transition probability matrix was parsimoniously determined using DAR. Each row of the matrix Q consisted of the negativebinomial probabilities of the process $\left\{X_{i} / 50\right\} \quad$ (refer to Section 3.4 on page 10). The final model requires a total of ten parameters, which we summarize in Table 2.

Table 2. Process summary of hierarchical model.

\begin{tabular}{|l|c|c|}
\hline \multicolumn{1}{|c|}{ Process Name } & Process Type & Parameters \\
\hline Scene Length & i.i.d & $\alpha, \lambda$ \\
\hline Number of Cells in Scene Change Frame & i.i.d & $\alpha, \lambda$ \\
\hline Number of Cells in next Frame after Scene Change Frame & deterministic & $a, b, \sigma^{2}$ \\
\hline Number of Cells in Frame within a Scene & correlated & $\rho, r, p$ \\
\hline
\end{tabular}

Frater, et al. [7] proposed a similar model; however, the scene change boundaries were determined in a slightly different way and processes for the first two frames after a scene change were not considered. Scene change boundaries were determined by detecting a large difference in the output of combination median, averaging filters. The scene length pdf was found to be of the form

$$
p(x)=\frac{a}{x^{n}+b^{2}}
$$

where $a$ and $b$ are constants, and $n$ is estimated from the empirical sequence.

This model also used the DAR process and the negative-binomial distribution for the frame size process; thereby, requiring a total of five parameters $(\rho, r, p, b, n)$. Even though the scene change frame process was not modeled, cell loss simulation results did appear to match well the results produced when using empirical trace data. 


\section{Models Based on the TES Process}

TES processes are designed to fit simultaneously both the marginal distribution and the autocorrelation function of the empirical data. It is a general method in that it can match any marginal distribution exactly and simultaneously approximate a wide variety of autocorrelation functions. A software package called TEStool supports TES modeling via a graphical user interface which facilitates both algorithms and interactive searches for TES models [14]. Recently, Jelenkovic and Melamed [23] have developed an algorithm which largely automates the search process and invariably leads to a more accurate model than those obtained via human interaction.

TES's ability to match marginal distributions exactly and approximate many autocorrelation functions makes it an excellent choice for constructing a source model. We give a brief overview of TES processes; however, for more in-depth information, the reader is referred to [35].

\subsection{Overview of TES Process}

TES defines a method for generating an auxiliary background process, $\left\{U_{n}\right\}$, which allows one to vary the nature of dependence among the target random variables $\left\{X_{n}\right\}$. The process $\left\{X_{n}\right\}$ is referred to as the foreground process and is generated from $\left\{U_{n}\right\}$ by using a suitable transformation. The process $\left\{U_{n}\right\}$ defines a random walk on the unit circle (circumference 1) based on the modulo-1 (fractional part) operator, defined as $\langle x\rangle=x-\lfloor x\rfloor^{10}$.

10. $\lfloor x\rfloor$ is the largest integer less than or equal to $\mathrm{x}$ (floor function). 
Specifically, TES background processes come in two flavors, $\left\{U^{+}\right\}$and $\left\{U_{n}^{-}\right\}$, defined as,

$$
U_{n}^{+}=\left\{\begin{array}{ll}
U_{0}, & n=0 \\
\left\langle U_{n-1}^{+}+V_{n}\right\rangle, & n>0
\end{array} \quad U_{n}^{-}= \begin{cases}U_{n}^{+}, & \text {n even } \\
1-U_{n}^{+}, & n \text { odd }\end{cases}\right.
$$

where the initial value $U_{0}$ is uniformly distributed on the interval $[0,1)$ and $\left\{V_{n}\right\}$, called the innovation sequence, consists of a sequence of i.i.d. random variables which are independent of $U_{0}$. The background process $\left\{U^{+}\right\}$can generate both negative decaying or oscillatory autocorrelation functions while $\left\{U^{-}\right\}$generates autocorrelations which alternate in polarity between odd and even lags.

In general, $\left\{V_{n}\right\}$ is obtained from the innovation density $f_{V}$ which is typically restricted to step-functions in order to simplify the parameter search. In the simplest non-trivial case of uniform innovations, $\left\{V_{n}\right\}$ is determined by two parameters $L$ and $R$ where

$$
V_{n}=L+(R-L) Z_{n}
$$

is generally referred to as the single-step innovation function, $Z_{n}$ are i.i.d. and uniformly distributed on the interval $[0,1)$ and $-0.5 \leq L<R<0.5$. The parameterization $(L, R)$ is equivalent to the parameterization $(\alpha, \phi)$ where

$$
\begin{aligned}
& \alpha=R-L, \\
& \phi=\frac{R+L}{R-L} .
\end{aligned}
$$

The $(\alpha, \phi)$ parameterization is convenient for calculating the autocorrelation function. The parameter $\alpha$ controls the magnitude of the autocorrelation function and $\phi$ controls its oscillations.

Once the background process is determined, the next step is to define the desired foreground process $\left\{X_{n}\right\}$. This is done by applying a transformation called the distortion function, to $\left\{U_{n}^{+}\right\}$or $\left\{U_{n}^{-}\right\}$. A common distortion is of the form $D=H_{Y}^{-1} \circ S_{\xi}$, where $H_{Y}^{-1}$ is 
the inverse of the cumulative histogram of the empirical data and $S_{\xi}$ is a stitching transform. A stitching transformation is used, parameterized by a stitching parameter $0 \leq \xi \leq 1$ where

$$
S_{\xi}(y)=\left\{\begin{array}{cc}
\frac{y}{\xi} & 0 \leq y \leq \xi \\
\frac{1-y}{1-\xi} & \xi \leq y<1
\end{array}\right.
$$

$S_{\xi}$ is an intermediate step designed to "smooth" the sample paths of $\left\{U_{n}^{+}\right\}$and $\left\{U_{n}^{-}\right\}$ when crossing the origin. In most cases, a value at or near 0.5 is selected for $\xi$. Finally, the requisite foreground TES sequence is defined by,

$$
X_{n}=H_{Y}^{-1}\left(S_{\xi}\left(U_{n}\right)\right) \text {. }
$$

\subsection{H.261 GOB video conference model using a TES process}

Melamed, et al. [37] developed a model for the number of bits per group-of-blocks (GOB) for H.261-encoded video. In H.261, each frame (352x288 pixels, Common Interface Format, CIF) consists of 12 GOBs, each containing 33 Macroblocks. A Macroblock contains four luminance blocks (block $=8 \times 8$ pixels) and two chrominance blocks. The simulated system was an 802.3 LAN driven by multiple video sources. Each data packet consisted of one or more GOBs.

They observed that the bit-rate data, at the GOB level, contained a significant periodic component. The reason for this is that each GOB retains significant correlations between the same blocks in successive frames since a block encodes the same portion of a frame and scene content changes slowly over time (seconds). They removed this component from the sample data, and then applied TES to the residual process, $R_{n}$. The periodic com- 
ponent was determined by using periodogram analysis to estimate the parameters $K, \omega_{\mathrm{i}}, A_{\mathbf{i}}$ and $B_{i}$. These parameters are used to model the periodic component as

$$
P_{n}=\sum_{i=1}^{K}\left(A_{i} \cos \omega_{i} n+B_{i} \sin \omega_{i} n\right)
$$

The residual process is then,

$$
R_{n}=X_{n}-P_{n}
$$

A single-step innovation density with $\alpha=0.50$ and $\phi=0.30$ was determined for $R_{n}$ (using the TEStool software) and the stitching parameter, $\xi$, was set to 0.5 . The periodic process was added to the TES process yielding the final foreground process

$$
X_{n}=H_{Y}^{-1}\left(S_{0.5}\left(U_{n}^{+}\right)\right)+P_{n}
$$

The parameters for the model are then $\left\{\alpha, \phi, \xi, A_{i}, B_{i}\right\}$ where $i=1,2 \ldots K$. The model produced an autocorrelation which matched its empirical data counterpart up to a lag of 100 frames. It then was compared to an earlier AR model which did not extract the periodic component, but distributed the bits within a frame equally across GOBs. Simulation showed the TES model produced lower packet loss and delay given the same throughput when compared to results using the AR model.

\subsection{Frame and Slice layer models using a generalized TES (G-TES) process}

Lazar et al. [26] developed both a frame and slice layer models for a DCT encoded version of the movie Star Wars. They used a generalized TES process in which the innovation process is not i.i.d. Each scene was modeled as a stationary process and scene lengths followed a geometric distribution with parameter

$$
p=\frac{1}{1+E\left[L_{n}\right]},
$$


where $E\left[L_{n}\right]$ is the expected value of the duration of a scene, $L_{n}$. Scene change boundaries were determined using the absolute difference in bit-rates of adjacent frames. The model attempts to capture the large change in bit-rate magnitude observed at scene change boundaries. The scene change process was incorporated into the innovation process as

$$
V_{n}=\left(1-W_{n}\right)\left(L+(R-L) Z_{n}\right)+W_{n}\left(-\frac{\alpha_{c}}{2}+\alpha_{c} Z_{n}\right)
$$

where $\left\{Z_{n}\right\}$ is a sequence of i.i.d. random variables with uniform marginals in the interval $[0,1)$, and $\left\{W_{n}\right\}$ is a sequence of i.i.d. Bernoulli random variables signaling that a scene change occurred. The GTES parameters were determined heuristically to be $\alpha_{c}=0.28$ and $R=-L=0.001$. The average scene length, $E\left[L_{n}\right]$, was found to be about 100 frames.

The same innovation process, with only a slight modification, was used for the slice layer model. In order to replicate the pseudo-periodic behavior of the autocorrelation function, they added a modulating function $a_{n}$. The background process was then defined as

$$
U_{n}=\left\langle U_{n-1}+a_{n} V_{n}\right\rangle
$$

where

$$
a_{n}= \begin{cases}1 & \text { if } 0 \leq n \% s \leq \frac{s}{2}-1 \\ -1 & \text { if } \frac{s}{2} \leq n \% \mathrm{~s} \leq s-1\end{cases}
$$

with $s$ being the number of slices per frame and $\%$ is the modulo operator.

The value of $E\left[L_{n}\right]$ must now be expressed in terms of slices, so a scale transformation changed it from 100 frames to 3000 slices (30 slices per frame). The parameters $L$ and $R$ were set to 0.003 and 0.008 respectively, while $\alpha_{c}$ was left intact.

The final model for both the frame and slice layer was

$$
X_{n}=H_{Y}^{-1}\left(S_{\xi}\left(U_{n}\right)\right)
$$

where $a_{n}=1$ for the frame layer model, and $a_{n}$ is given by (37) for the slice layer model. 
The parameters for the model are $\left\{\alpha_{s} \alpha_{c}, \xi, p\right\}$ for the frame layer and $\left\{\alpha_{s} \alpha_{c}, \xi, p, a_{n}\right\}$ for the slice layer. The frame layer model matched the autocorrelation function of the empirical data well up to a lag of about 300 frames, but thereafter it dropped below its empirical counterpart. The slice layer model matched the autocorrelation well up to a lag of 100 frames. The pseudo-periodic behavior was captured, but the model appeared to underestimate the peaks in the autocorrelation function. The attractiveness of the GTES approach, is that it provides a way to model VBR video at two different time scales (slice and frame), while directly incorporating a scene change mechanism. This is the first model considered to be of the hierarchical type. The model was used in simulations to determine the bandwidth allocation required for sources of different service types.

\subsection{MPEG Frame layer model using a composite-TES (C-TES) processes}

Reininger, et al. [42] developed a model for MPEG sequences containing $I, B$ and $P$ frames. The model uses the background process $\left\{U^{+}\right\}$for $I, B$ frames and $\left\{U^{-}\right\}$for $P$ frames giving the background sequences, $\left\{U^{I+}\right\},\left\{U^{B+}\right\}$ and $\left\{U^{P-}\right\}$. The scene change process was not taken into account in this model.

An interesting point regarding this model is that the number of bits in $B$ and $P$ frames, within a Group Of Pictures (GOP), depends on the $I$ frame located at the beginning of a GOP. Recall that in MPEG, a GOP consists of a set of $I, B$ and $P$ frames arranged in a deterministic pattern which repeats throughout the sequence. For example, given a frame sequence $\left\{\ldots I_{2} B_{3} B_{4} P_{2} B_{5} B_{6} P_{3} B_{7} B_{8} I_{3} \ldots\right\}$ where the subscript is the index number of the associated frame type, the background sequence variates for $B_{3}$ and $P_{2}$ are set equal to the background sequence variate for $I_{2}\left(U_{2}^{I+}=U_{3}^{B+}=U_{2}^{P-}\right)$. This makes intuitive sense, because both B and P frames, within a GOP, reference the I frame in the encoding process. An illustration of the relationship of the background sequences for $I, P$ and $B$ frames is shown in Figure 4. 


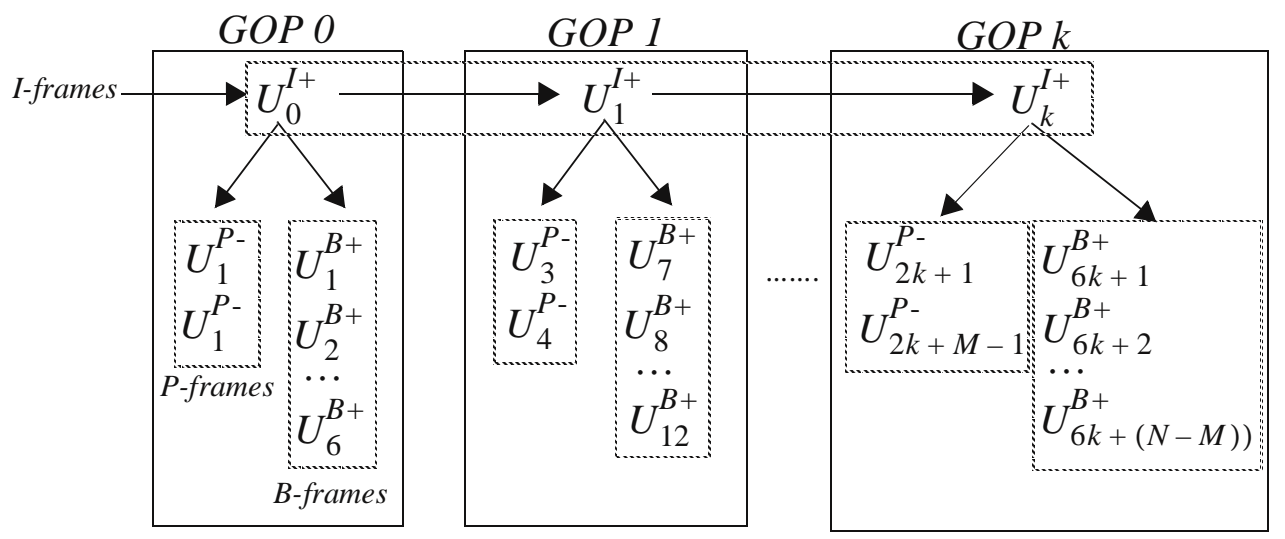

Figure 4. Generation of background processes for I, $P$ and $B$ frames in relation to the GOP sequence.

The final model is composed of a deterministic combination of each frame type process. Process selection is determined by the GOP frame sequence pattern, defined by the MPEG encoding parameters $N$ and $M$, which are the I frame and $\mathrm{P}$ frame distances respectively. The three random processes are defined as,

$$
\begin{aligned}
& X_{n}^{I+}=H_{Y_{I}}^{-1}\left(S_{\xi_{I}}\left(U_{n}^{I+}\right)\right), \\
& X_{n}^{B+}=H_{Y_{B}}^{-1}\left(S_{\xi_{B}}\left(U_{n}^{B+}\right)\right), \\
& X_{n}^{P-}=H_{Y_{P}}^{-1}\left(S_{\xi_{P}}\left(U_{n}^{P-}\right)\right) .
\end{aligned}
$$

The model requires nine parameters, $\{\alpha, \phi, \xi\}_{I, B, P}$, three for each frame type. One point of interest here is that MPEG sequences with IBP frames exhibited a pseudo-periodic autocorrelation function similar to that produced by slice layer sequences. This behavior is caused by the deterministic sequencing of IBP frames and is captured well by this model.

\subsection{Frame layer model using a Markov modulated TES (MRMT) process}

Melamed and Pendarakis [36] developed a model for a DCT encoded version of the movie Star Wars (this is the same sequence used in Section 4.3) and takes scene changes into account. The approach taken here is different from that in Section 4.3 in that the scene 
change process is not assumed to be i.i.d, but Markovian. The first task was then to segment the video sequence into individual scene segments and classify each segment. Scene boundaries were detected by measuring the sustained absolute difference of the bit-rates between a series of successive frames, again, similar to the technique was used in Section $4.3^{11}$.

Once the video sequence is segmented, each segment is classified using a clustering algorithm based on the minimum Euclidean distance between mean bit-rates. Four clusters were sufficient to categorize the video sequence into four scene classes. A TES model was then created for each scene class where each class was mapped to a state of a Markov Chain, illustrated in Figure 5, whence the name Markov Renewal Modulated TES (MRMT) process. Scene durations were class dependent; however, its autocorrelation function was practically zero for lags greater than five frames, so it was assumed to be i.i.d. random variable, represented by $\left\{T_{i}\right\}$, where $i=1,2,3,4$ is the class index. Although it is not explicitly stated that the scene duration distribution is geometric, the video sequence is the same as that used in [26] where its form is stated explicitly.

The final model consists of four TES processes, one for each class,

$$
X_{n}^{i+}=H_{Y_{i}}^{-1}\left(S_{\xi_{i}}\left(U_{n}^{i+}\right)\right) \quad i=1,2,3,4
$$

as well as four renewal processes for the corresponding scene durations.

The parameters for the model are $\left\{\alpha_{i} \phi_{i}, \xi_{i}, P\right\}$, where $i$ is the class index and $P=\left[p_{i, j}\right]$ transition probability matrix. This yields 12 parameters for the matrix, and 3 for each TES process giving a total of 24 parameters. The model was used to generate 171,000 samples and the attendant sample path, histogram, autocorrelation function and spectral density were compared to their empirical counterparts. In all cases excellent

11. It should be noted, that sequences containing I, B and P frames will require different methods to determine scene change boundaries. It seems feasible that, in this case, this technique could be applied to I frames only. 


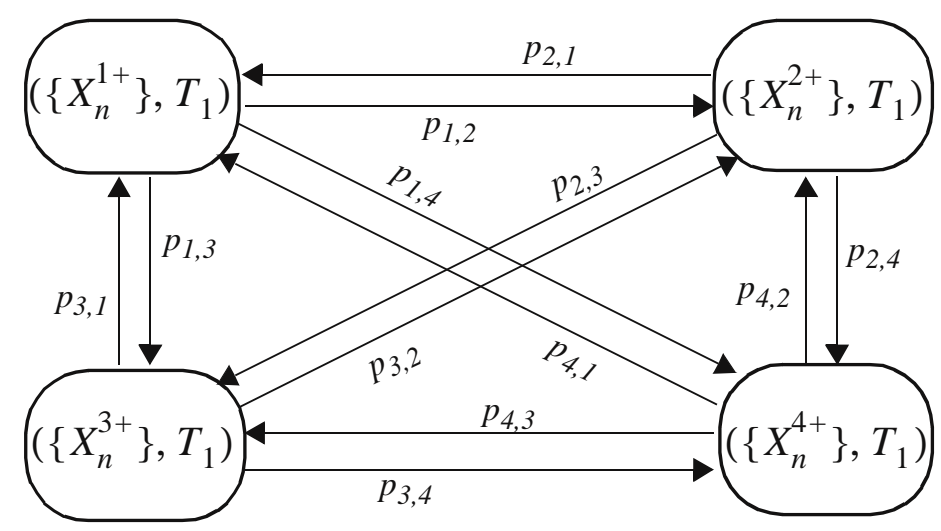

Figure 5. The modulating Markov Chain which controls TES process selection and scene duration based on class type. Transition probabilities, $p_{i}$, from class $i$ to class $j$ are determined from the empirical data of scene transitions. The amount of time spent in a particular state, $T_{i}$, is sampled from the empirical distribution of class $i$ scene durations. $\left\{X_{n}^{i+}\right\}$ represents the TES process for scene class $i$.

matches were achieved. This model produced better matches to the autocorrelation function at long lags since the Markov Chain captures the longer-term scene change behavior. 


\section{Models Based on Self-Similar Processes}

Loosely speaking, a process is said to be self-similar if the samples for that process appears "similar", regardless of the duration of the sampling interval (time scale). One of the important characteristics of a self-similar processes is that it is long-range dependent (LRD) which means that: (1) The autocorrelation function is not summable, (2) The power spectrum at low frequencies is unbounded and approaches infinity as the frequency approaches zero (DC component). This behavior differs from short-range dependent (SRD) processes whose autocorrelation functions are summable and power spectrums are bounded at low frequencies. Recent work by Beran, et al. [2] has shown that long-range dependence is intrinsic to VBR sequences, and given that there is some evidence that LRD processes can negatively affect multiplexing performance [1,30], source models which capture this characteristic were developed. We present a brief overview of LRD; however, for more information the reader should see [2].

\subsection{Overview of Long-Range Dependence}

It has been found that LRD processes occurs quite often in nature. Natural phenomena such as rainfall, the annual growth of tree rings, river levels and discharges are often described as self-similar processes. Hurst first discovered this property by investigating the amount of storage required in the Great Lakes of the Nile river basin [17]. He found that the expected value of the quantity $R(n) / S(n)$ (rescaled adjusted range statistic $R /$ $S)$, asymptotically followed a power law

$$
E[R(n) / S(n)] \approx c n^{H}, \quad n \rightarrow \infty
$$


where $c$ is a positive constant independent of $n, R(n)$ is the adjusted range, $S(n)$ is the sample standard deviation, and $H$ is the Hurst Parameter with range $0.5<H<1$. The rescaled adjusted range is calculated using

$$
\frac{R(n)}{S(n)}=\frac{\max \left(0, W_{1}, W_{2}, \ldots W_{n}\right)-\min \left(0,0, W_{1}, W_{2}, \ldots W_{n}\right)}{S(n)}
$$

where

$$
\begin{gathered}
W_{0}=0, \\
W_{k}=\sum_{i=1} X_{i}-k \bar{X}(n) \quad k=1,2, \ldots, n
\end{gathered}
$$

and $X_{i}$ is the empirical sequence.

The value $E[R(n) / S(n)]$ is calculated for different values of $n$ and plotted in a Pox diagram where $\log (E[R(n) / S(n)])$ is plotted on the y axis and $\log (n)$ is plotted on the $\mathrm{x}$ axis. Linear regression is then used to estimate the Hurst parameter where

$$
H=\frac{\log E[R(n) / S(n)]}{\log n} .
$$

Typical values of $H$ for various sequences are given in Table 3. Note that the value of $H$ for i.i.d random processes is 0.5 , while for computer traffic it is approximately 1 . Figure 6 shows the effect of the value of $H$ on a random sequence. We plotted samples generated using a fractional ARIMA process for four different values of $\mathrm{H}$. We see that as $H$ increases, a noticeable low frequency oscillation is evident in the sequence envelope.

Once $H$ is estimated, a processes such as Fractional ARIMA, or Fast Fractional Gaussian Noise (ffGn) is used to create a background sequence, which may be used in turn to generate the foreground sequence using the desired empirical marginal bit-rate distribution. Other methods can be used to estimate $H$ in addition to R/S analysis such as Variance-Time and Periodogram analysis. 

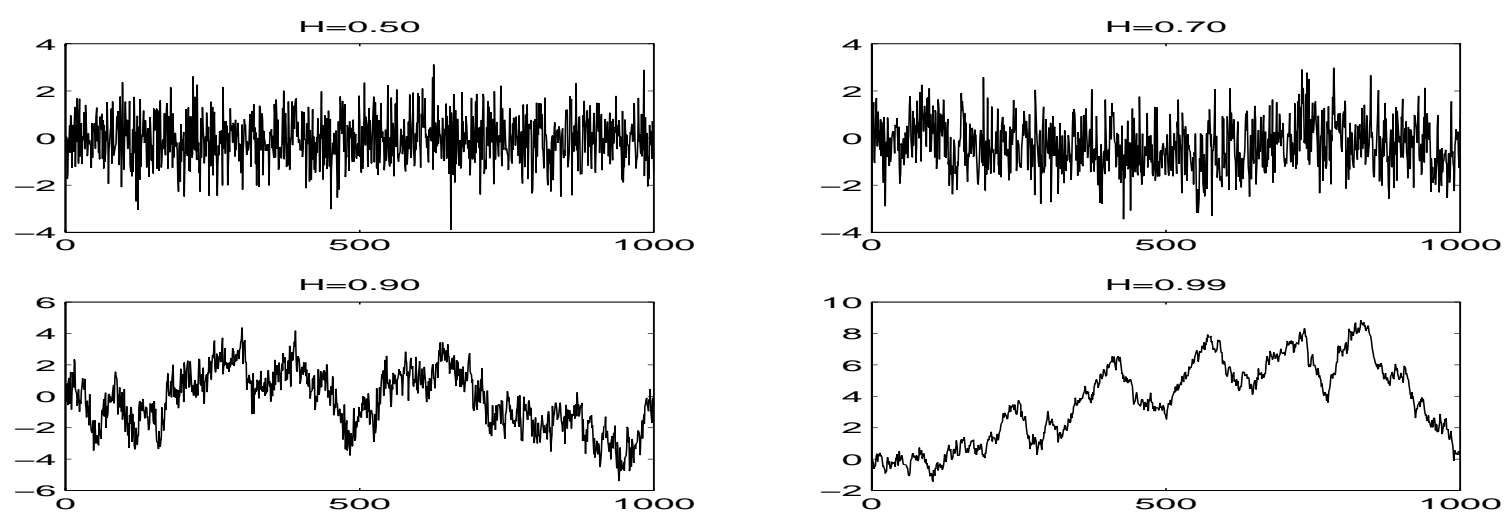

(a)
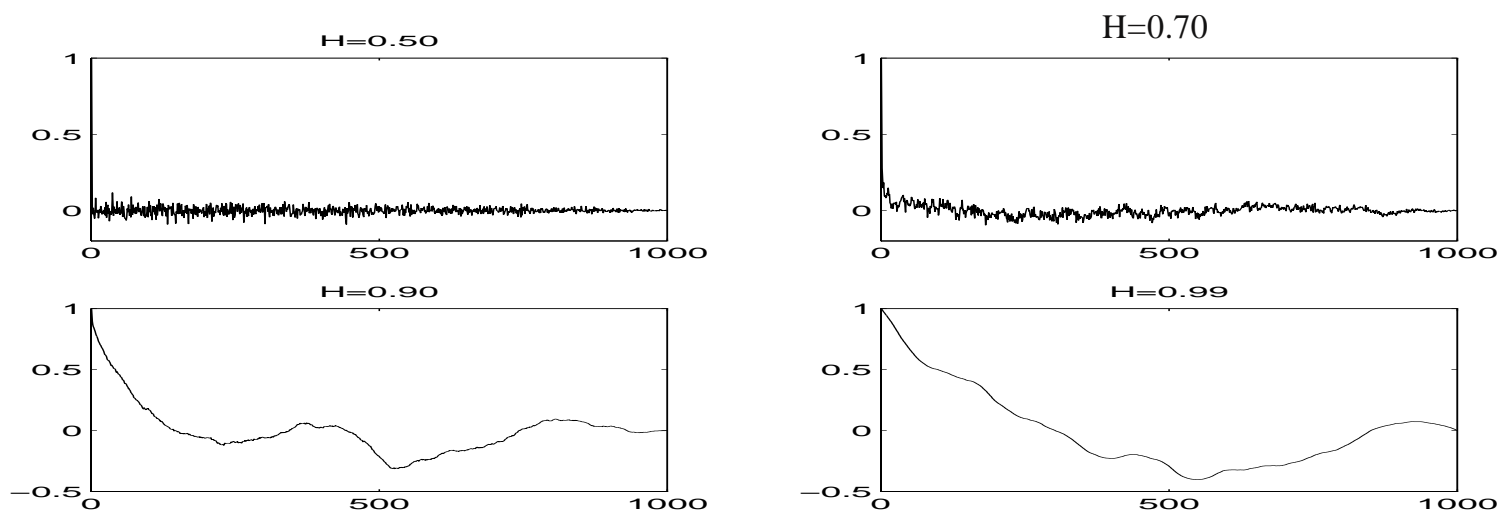

(b)

Figure 6. Comparison of the Hurst effect on random process (a) samples generated using fractional ARIMA process $(x$-axis $=$ sample, $y$-axis $=$ magnitude $)(b)$ corresponding empirical autocorrelation functions ( $x$-axis = correlation coefficient, $y$-axis $=\mathbf{l a g})$.

Table 3. Hurst parameters of various traffic types.

\begin{tabular}{|l|l|}
\hline \multicolumn{1}{|c|}{ TRAFFIC TYPE } & \multicolumn{1}{c|}{ H } \\
\hline Computer Traffic [29] & $\approx 1$ \\
\hline CNN [4] & 0.90 \\
\hline Star Wars (Motion JPEG) [4] & 0.88 \\
\hline Star Wars (MPEG IBP) [4] & 0.86 \\
\hline Star Wars (B/W, DCT) [8] & 0.83 \\
\hline Video Conference [2] & $0.6-0.75$ \\
\hline i.i.d [6,33] & 0.5 \\
\hline
\end{tabular}

\subsection{Frame layer model using a fractional ARIMA (f-ARIMA) process}

Garrett and Willinger [8] developed a model a DCT encoding of the movie Star Wars (same one used in Section 4.3). To match both the left and right tails of the empirical dis- 
tribution they used a hybrid distribution, $F_{\Gamma / P}$, which consisted of a concatenation of a Gamma distribution for the left tail and a Pareto distribution for the right tail. Right tail matching is particularly important because it describes the probabilities of high bit-rates which can significantly affect queueing performance.

The model uses a fractional $\operatorname{ARIMA}(0, d, 0)$ process to generate the background sequence, where $d=H-0.5$, and its autocorrelation function is given by

$$
\rho_{k}=\frac{d(1+d) \ldots(k-1+d)}{(1-d)(2-d) \ldots(k-d)}
$$

The background process, $\left\{U_{k}\right\}$, is generated using Hosking's algorithm ${ }^{12}[15]$ which is an $o\left(n^{2}\right)$ algorithm. Each $U_{k}$ is Gaussian with mean $\mu_{k}$ and variance $\sigma_{k}^{2}$ which are given by the function $\phi$, defined recursively by

$$
\begin{gathered}
\phi_{k j}=\phi_{k-1, j}-\phi_{k k} \phi_{k-1, k-j}, \quad j=1, \ldots, k-1 \\
\phi_{k k}=\left(N_{k} / D_{k}\right),
\end{gathered}
$$

where

$$
\begin{gathered}
N_{k}=\rho_{k}-\sum_{j=1}^{k-1} \phi_{k-1, j} \rho_{k-j}, \\
D_{k}=D_{k-1}-\left(N_{k-1}^{2} / D_{k-1}\right) .
\end{gathered}
$$

and the initial values are $N_{0}=0$ and $D_{0}=1$. The mean and variance is then

$$
\sigma_{k}^{2}=\left(1-\phi_{k k}^{2}\right) \sigma_{k-1}^{2}
$$

12. One interesting feature of this algorithm is that, given a discrete autocorrelation sequence, $\rho_{k}$, a sample path can be generated whose autocorrelation matches it. We will see this technique used later in a model proposed by Huang 


$$
\mu_{k}=\sum_{j=1}^{k} \phi_{k j} X_{k-j},
$$

where the random variable, $X_{0}$, is sampled from a standard normal distribution. Finally, the number of bits per frame is represented by the foreground sequence, $\left\{Y_{k}\right\}$, which is generated using the transformation

$$
Y_{k}=F_{\Gamma / P}^{-1}\left(F_{N}\left(U_{k}\right)\right) . \quad k>0
$$

where $F_{N}$ is the standard normal cumulative distribution function and $F_{\Gamma / P}^{-1}($.$) is the$ inverse of the aforementioned hybrid Gamma/Pareto cumulative distribution function. Note that the LRD property is still preserved when transforming from $U_{k}$ to $X_{k}$.

The parameters required by the model are $\left(\mu_{\Gamma}, \sigma_{\Gamma}, m_{T}, H\right)$ where $\mu_{\Gamma}$ and $\sigma_{\Gamma}$ are the mean and variance of the Gamma distribution and $m_{T}$ is the slope of the line which best fits the tail of the Pareto distribution. The model parsimoniously captures the LRD aspect in this sequence using a single parameter.

\subsection{Frame layer model using a fast fractional Gaussian Noise (ffGN) approximation}

Enssle [5] developed a VBR model for an MPEG version of Star Wars containing I,B and $P$ frames. The model used the fast fractional Gaussian Noise (ffGN) algorithm to generate the background sequence. This method is an approximation to the fractional Gaussian noise process and has a computational complexity of $o(n)$. Consequently, $f f G N$ provides a faster way to generate the background sequence as compared to fractional ARIMA.

The background process, $\left\{U_{s}(t)\right\}$, was generated using $f f G N$. The Hurst parameter, $H$, was estimated to be 0.856 , using Periodogram and $R / S$ analysis, which compares well with the value 0.83 found in [8]. The $f f G N$ algorithm requires two additional parameters besides $H$, called the base, $B$, and quality, $Q$. Selecting a value for $B$ in the range $1.1<B<2$ and $\mathrm{Q}=20$ produced acceptable estimates of the autocorrelation function 
(This was heuristically determined). The background process, $\left\{U_{s}(t, H)\right\}$, consists of high and low frequency components,

$$
U_{s}(t, H)=U_{l}(t, H)+U_{h}(t, H) .
$$

The low frequency component is a weighted sum of independent Markov-Gauss processes,

$$
U_{l}(t, H)=\sum_{k=1}^{N} W_{k} G\left(t, r_{k}\right),
$$

The number of Markov-Gauss processes is

$$
N=\lceil\ln (Q n) / \ln (B)\rceil,
$$

where $n$ is the length of the time series and the weight factors are

$$
W_{k}=\sqrt{\frac{H(2 H-1)\left(B^{1-H}-B^{H-1}\right)\left(B^{-2 k(1-H)}\right)}{\Gamma(3-2 H)}} .
$$

The lag-1 covariance is given by

$$
r_{k}=e^{-B^{-k}}
$$

which is used in the Markov-Gauss process

$$
G\left(t, r_{k}\right)=\left\{\begin{array}{cc}
G_{k}(1), & t=1 \\
r_{k} G\left(t-1, r_{k}\right)+\sqrt{\left(1-r_{k}^{2}\right)} G_{k}(t), & t>1
\end{array}\right.
$$

where $G_{k}(t)$ is standard normal. 
The high frequency component of (53) is

$$
U_{h}(t, H)=\sqrt{1-\frac{B^{H-1}}{4(1-H) \Gamma(-2 H)}} G(t)
$$

where $G(t)$ is standard normal.

Each frame type has an corresponding distribution for the number of bits per frame, which was determined to fit the Lognormal distribution well. The background process, $U_{s}(t, H)$, is then distorted using the transformation of the frame type distribution, $F_{i}^{-1}$, to give

$$
X_{i}(t)=F_{i}^{-1}\left(U_{s}(t)\right), \quad i=1,2, \ldots, n
$$

which translates to the following

$$
X_{i}(t)=\exp \left[\sqrt{\ln (1+\Phi)} X_{s}(t)+\ln E\left[X_{i}\right]-\frac{1}{2} \ln (1+\Phi)\right]
$$

where $\Phi=\left(\operatorname{Var}\left[X_{i}\right]\right) / E\left[X_{i}\right]^{2}$ and $i$ is the frame type $I, B$ and $P$.

The model requires three parameters: $B, Q$ and $H$ for the background process and six parameters: $E\left[X_{i}\right], \operatorname{Var}\left[X_{i}\right]$, for the Lognormal distributions, for a total of nine parameters. The model basically operates as follows. At time $t$, the frame type $i$, which is predetermined by the MPEG GOP pattern (IBBPBBP...), is used to select one of the three transform functions: $X_{I}(t), X_{B}(t)$ and $X_{P}(t)$. A sample is then generated of $\left\{U_{s}(t, H)\right\}$ which is used in the selected transform function. Time is then incremented to determine the next frame type and the process continues for the duration of the video sequence. 


\subsection{Frame layer SRD/LRD model using a f-ARIMA process}

Huang, et al. [16] developed a model for an MPEG encoding of the movie Last Action Hero, which contains I, B and P frames. It is referred to as a unified model because it uses a hybrid function in order to match the autocorrelation function at both the short-term and long-term lags. The Hurst parameter, $H$, was used to generate the LRD part of the autocorrelation function, and a weighted sum of exponentials was used to match the SRD part. These parts were then combined yielding the hybrid autocorrelation function

$$
\rho_{k}= \begin{cases}e^{-0.00565 k} & k<K_{t} \\ 1.59 k^{-0.2} & k \geq K_{t}\end{cases}
$$

where $K_{t}$ is the boundary between the SRD and LRD parts. The Hurst parameter was determined using both R/S and Variance-time analysis and the SRD parameters were determined by using regression analysis. Once the autocorrelation function was determined, Hosking's algorithm was used to define the background process, $\left\{U_{n}\right\}$. Individual histograms were constructed for each frame type and the attendant background process was then transformed for each frame type via

$$
X_{n}^{i}=H_{Y}^{-1}\left(U_{n}\right) . \quad i=I, B, P .
$$

Histograms were used since the authors determined that the empirical distributions did not seem to fit any of the existing classical distributions.

The model requires four parameters to estimate $\rho_{k}$, the Hurst parameter $H$, and one parameter for each cell in the frame type histogram. Samples were produced which matched the autocorrelation function well for lags up to 500 frames. The portion of the autocorrelation function up to lag $K_{t} \sim 75$ is fitted well by the exponential function. This is probably the most accurate model to date in terms of matching the autocorrelation function for both short and long lags. 


\section{Analytical and non-Markovian type models}

The analytical model proposed by Marafih, et al. [34] is a model whose premise relies on a discrete-state Markov Chain and uses analytical methods such as: stationary-interval (SI), asymptotic method (ASM) and Queueing Network Analyzer (QNA) in order to estimate multiplexer cell loss. Model parameters were estimated using the first two moments of the empirical sequence (I and P frames). Results showed that the SI model produced results which provided an good upper bound while the ASM model provided a lower bound. The QNA model seemed to match the results using the empirical data.

Skelly, et al. [44] proposed a generalized histogram-based analytical model for ATM VBR sources. Each source is characterized by a bit-rate histogram and the histogram of the aggregate (multiplexed) process is obtained by convolving the individual source histograms. Once obtained, the histogram is used to solve an $\mathrm{M} / \mathrm{D} / 1 / \mathrm{K}$ system and the buffer occupancy distribution is given by

$$
P(n)=\sum_{I=1}^{N} P\left(n \mid \lambda_{I}=\lambda\right) P\left(\lambda=\lambda_{I}\right)
$$

where $P\left(\lambda=\lambda_{I}\right)$ is the bit-rate histogram approximation of $f_{\lambda}(x), P\left(n \mid \lambda=\lambda_{I}\right)$ is the buffer occupancy distribution given the arrival rate is $\lambda_{I}$, and $N$ is the number of bins in the histogram. In order to generalize the applicability of their model, they used an $M M P P / \mathrm{E}_{\mathrm{k}} / 1 / \mathrm{K}$ as the source model to determine the buffer occupancy distributions. They found that a Markov Chain of eight states was sufficient; thereby, requiring an 8x8 transition probability matrix. This model requires 64 parameters for the matrix and 8 parameters for the average arrival rate for the Poisson process in each state, for a total of 72 parameters.

Krunz, et al. [25] proposed a model for an MPEG encoding of the movie The Wizard of $O z$ consisting of 41,760 frames (I,B and P). Each frame type was fitted using a Lognormal distribution, where parameters were estimated using the Maximum Likelihood Estimators (MLE). The frame size process for each frame type was assumed to be i.i.d which greatly simplifies the model. Selection of the appropriate frame type processes was done 
deterministically based on the MPEG GOP structure. While this model is simplistic, simulation results for cell loss, when multiplexing up to 10 sources, matched the results produced by the empirical sequence fairly well. A similar model was proposed by Enssle [4] for a full-length (123,574 frames) MPEG encoding of the movie Star Wars. 


\section{Summary}

It is apparent that one has many choices when it comes to VBR models. In order to narrow the selection, it is important that the user define the attributes of the source in order to assist in model selection. Some questions to answer are:

1. Does the VBR sequence contain significant scene changes?

2. What is the frame type sequence pattern? Is it H.261 or MPEG?

3. Does the sequence contain B frames?

4. Does the frame type distribution fit any of the classical distributions, such as Lognormal, Gamma, etc.?

5. What level is to be modeled? Frame, slice, GOB, or ATM cell?

Answering these questions will assist the user in determining the proper VBR model to use in simulations. A sequence which contains different frame types (IBP) would likely require a model which uses separate processes for each frame type, so this is an important consideration. Another matter to consider is the marginal distribution of the source process. If a classical distribution will fit, then samples can be generated using many of the existing mathematical transformations; otherwise, samples must be generated using the inversion method on the empirical histogram. We summarize the attributes of the models covered in this survey in Table 4.

Table 4. Summary of VBR model attributes.

\begin{tabular}{|l|l|c|c|c|c|}
\hline$\#$ & \multicolumn{1}{|c|}{ Model Type } & Parameters & $\begin{array}{c}\text { Scene } \\
\text { Change? }\end{array}$ & Level & $\begin{array}{c}\text { B } \\
\text { Frames? }\end{array}$ \\
\hline 1 & ARMA [9] & 1003 & No & sub-frame & No \\
\hline 2 & DAR $^{\mathrm{a}}[13]$ & 4 & No & Frame & No \\
\hline 3 & MRP [27] & 40 & No & Frame & No \\
\hline 4 & AR/MC [41] & 8 & Yes & Frame & No \\
\hline 5 & MC [39] & $51-102$ & No & Frame/Slice & No \\
\hline 6 & AR/MC [46] & 9 & No & Frame & No \\
\hline 7 & AR [22] & 20 & No & Block & No \\
\hline 8 & DAR/i.i.d [12] & 10 & Yes & Frame & No \\
\hline 9 & DAR/i.i.d. [7] & 5 & Yes & Frame & No \\
\hline \hline 10 & TES [37] & $2 \mathrm{~K}^{\mathrm{b}}+3+\mathrm{H}^{\mathrm{c}}$ & No & GOB & No \\
\hline
\end{tabular}


Table 4. Summary of VBR model attributes.

\begin{tabular}{|c|l|c|c|c|c|}
\hline$\#$ & \multicolumn{1}{|c|}{ Model Type } & Parameters & $\begin{array}{c}\text { Scene } \\
\text { Change? }\end{array}$ & Level & $\begin{array}{c}\text { B } \\
\text { Frames? }\end{array}$ \\
\hline 11 & GTES [26] & $5+\mathrm{H}$ & Yes & Frame/Slice & No \\
\hline 12 & composite-TES[42] & $9+3 \mathrm{H}$ & No & Frame & Yes \\
\hline 13 & MRMT [36] & $24+4 \mathrm{H}$ & Yes & Frame & No \\
\hline \hline 14 & f-ARIMA [8] & 4 & Yes & Frame & No \\
\hline 15 & ffGN [5] & 9 & Yes & Frame & Yes \\
\hline 16 & Unified f-ARIMA [16] & $4+3 \mathrm{H}$ & Yes & Frame & Yes \\
\hline \hline 17 & EGI/D/1 [34] & 2 & No & Frame & No \\
\hline 18 & MMPP/E $/$ //K [44] & 72 & No & Frame & No \\
\hline 19 & i.i.d [4] [25] & 6 & No & Frame & Yes \\
\hline
\end{tabular}

a. $\mathrm{MC}=$ Markov Chain

b. $\mathrm{K}=$ \# of frequency coefficients to match periodic GOB process.

c. " $\mathrm{H}$ " is number of histogram cells required by a TES process to match the empirical distribution.

\subsection{Model recommendations}

Several recommendations can be given, as a result of this survey, which might be helpful in determining an appropriate model for VBR video. The first is that if a video conference model is required (no B frames), and there are few or no scene changes, then a DAR model is a better choice than an AR model. Another contender, however, is the MRP model, which produced better results than DAR, but requires more parameters. It is conceivable, that for certain video sequences in which the bit-rate stays at particular levels for long periods of time that MRP would be a better choice.

Another recommendation deals with the modeling of the scene change process. When modeling sequences with numerous scene changes, the user can choose either a Markov based model or a self-similar model. The choice, however, is not clear-cut. On the one hand, self-similar models have the advantage that only a single parameter $(\mathrm{H})$ is required; whereas, a Markov Chain requires many parameters (transition probabilities). Unfortunately, the drawback to self-similar models is the computational complexity involved in 
generating samples can be quite high. This is alleviated somewhat by using $f f G N$. Nevertheless, it does appear from the results given in [8] and [5], that ignoring the scene change process will most likely yield overly optimistic simulation results.

The last recommendation deals with models for MPEG video which contain I, B and P frames. It seems clear that these models require separate processes for each frame type. The assumption to select each process deterministically according to the GOP pattern is valid. However, this is due to the fact that the GOP pattern is fixed for the duration of the sequence. Future encoders will most likely vary frame type sequencing based on video content which will vary the GOP pattern. In fact, this mode of operation is fully compliant with the MPEG standard. For such sequences, the frame type selection process will also be stochastic, thereby, invalidating the deterministic assumption. This opens a new area which is yet to be covered in VBR modeling.

\subsection{Self-Similar models}

One issue concerning self-similar models is the relative importance in matching the SRD component of the autocorrelation function. We saw that in Huang's model [16] both the SRD and the LRD components were matched. In effect, the background process exhibits both a rapid decay at short lags and a much slower decay for long lags. Enssle's model (ffGN) does not do this, but claims that the SRD component is sufficiently captured by the deterministic GOP process. A question which arises then is the added benefit derived in capturing the SRD component of an individual frame type. With regards to the question of the relative significance of the LRD component in VBR sequences, recent work by Heyman and Lakshman [11] suggests that short busy periods (typical when multiplexing VBR sequences) and finite buffers cause a "reset effect" which, for the most part, negates any LRD effects. As a consequence, they argue that SRD, which is modeled well by using Markov processes, is a more important aspect of VBR sequences. 


\subsection{Further research}

Most of the focus in VBR research has centered on packet loss at a multiplexer buffer. This metric is assumed to be directly related to video Quality of Service (QoS). However, aggregate loss might not be sufficient in determining end-user "perceived" quality. In fact, loss "bursts" and their frequency of occurrence might be a more significant metric. More specifically, VBR streams with similar aggregate loss, but significantly different mean time between losses (MTBL), might be perceived as having very different quality by the end user (This phenomena has been suggested in [8] and [43]). As a consequence, research is needed to determine how well useful these models are in predicting this metric.

Another direction for further work is the performance of a model when using a "smoother" mechanism. A smoother reduces the source traffic bit-rate peak-to-mean ratio by injecting extra delay. Models which do not accurately reproduce the empirical sample path might not be suitable when used in conjunction with a smoother. The only paper in this survey which partially addresses this issue is the one by Lucantoni, et al., where leaky bucket contour curves were studied. However, the leaky bucket is more of a traffic policing device rather than smoothing device and there are a whole class of smoothers which have not been studied in conjunction with any of the proposed models. In addition, the buffer service policy used by the multiplexer should be taken into account. Most of the models assume a First-Come-First-Serve policy, but others exist such as Weighted Fair Queueing.

Another area often overlooked (or minimized in its significance), is the cross-correlation effect between MPEG sequences which result from the GOP pattern. Sequences which have GOP parameters (N,M) which are the same, or multiples thereof, can have I and $\mathrm{P}$ frames which overlap in time. Also, since these frames are much larger than $\mathrm{B}$ frames, their overlap could cause serious degradations in multiplexer performance. Since the GOP pattern is deterministic, this overlap will continue for the duration of the video sequence. 
Finally, while it appears that a single model will not apply to all video sequences, a generalized modeling framework is required which can be applied to a wide variety of video content. It is not clear that this objective can be achieved using Markovian based models, since none have been applied to MPEG sequences containing B frames. As to whether a hierarchical model using Markov Chains and DAR can be used for such sequences, it remains to be seen. The two candidates found in this survey are the self-similar model, proposed by Enssle, and the MRMT model, proposed by Melamed and Pendarakis. Given the arguments by Heyman that the LRD aspect of VBR sequences is relatively unimportant when compared the SRD aspect, it would appear that a model such as MRMT can provide such a framework. 


\section{Acknowledgments}

We are grateful to Benjamin Melamed for his thorough and insightful reading of this manuscript. We would also like to thank Mark Garrett, Jurgen Enssle and Diane Gan for their helpful comments. 


\section{References}

1. A. Adas and A. Mukherjee, "On Resource Management and QoS Guarantees for Long Range Dependent Traffic,” IEEE INFOCOM, Vol 2, pp 779-787, 1995.

2. J. Beran, R. Shreman, M.S. Taqqu and W. Willinger, "Variable-bit-rate video traffic and long range dependence," IEEE Trans. on Commun., Vol 43, No 2-4, part 3, p1566-1579., Feb-Apr 1995.

3. CCITT Study Group XV, "Recommendation H.261," Recommendation of the HSeries, pp 79-107, August 1990.

4. J. Enssle, "Modeling and Statistical Multiplexing of VBR MPEG Compressed Video in ATM Networks," In Proc. 4th Workshop on High Speed Networks, Brest France, pp. 59-67, Sept. 7-9, 1994.

5. J. Enssle, "Modeling of Short and Long Term Properties of VBR MPEG Compressed Video in ATM Networks," Proceedings of the 1995 Silicon Valley Networking Conference \& Exposition, San Jose, Ca., pp 95-107, April 1995.

6. W. Feller, "The Asymptotic Distribution of the Range of Sums of Independent Random Variable," Ann. Math. Statists., Vol 22, pp. 427-432, 1951.

7. M. R. Frater, J. F. Arnold and P. Tan, "A New Statistical Model for Traffic Generated by VBR Coders for Television on the Broadband ISDN," IEEE Transactions on Circuits and Systems for Video Technology, Vol. 4, No. 6, pp 521-526, December 1994.

8. M.W. Garrett and W. Willinger, "Analysis, Modeling and Generation of Self-Similar VBR Video Traffic,” SIGCOMM, pp 269-280, 1994.

9. Rito Grunenfelder, John P. Cosmas, Sam Manthorpe and Augustine Odinma-Okafor, "Characterization of Video Codecs as Autoregressive Moving Average processes and Related Queueing System Performance," IEEE J. Sel. Areas Commun., Vol. 9 No. 3, pp. 284-293, April 1991.

10. B. Haskell, "Buffer and Channel Sharing by Several Interframe Picturephone ${ }^{\circledR}$ coders," The Bell System Technical Journal, Vol. 51, No. 1, pp 261-289, 1972.

11. D. Heyman and T. V. Lakshman, "What Are the Implications of Long-Range Dependence for VBR-Video Traffic Engineering?" IEEE/ACM Transactions on Networking, Vol. 4, No. 3, pp. 301-317, June 1996.

12. D. Heyman and T. V. Lakshman, "Source Models for VBR Broadcast-Video Traffic," Transactions on Networking, Vol. 4, No. 1, pp. 40-48, February 1996.

13. D. Heyman, A. Tabatabai, and T. V. Lakshman, "Statistical Analysis and Simulation Study of Video Teleconference Traffic in ATM Networks," IEEE J. Select. Areas Commun., vol. 2, no. 1, pp. 49-59, March 1992.

14. J.R. Hill and B. Melamed, "TEStool: A visual interactive environment for modeling autocorrelated time series," Performance Evaluation, Vol 24, pp. 3-22, 1995.

15. J.R.M.Hosking, "Modeling Persistence in Hydrological Time Series Using Fractional Differencing," Water Resources Res., Vol. 20, No. 12, pp.1898-1908, 1984. 
16. C. Huang, M. Devetsikiotis, I. Lambadaris and R. Kaye, "Modeling and Simulation of Self-Similar Variable bit-rate Compressed Video: A Unified Approach," ACM SIGCOMM, pp. 114-125, 1995.

17. H.E. Hurst, "Long-Term Storage Capacity of Reservoirs," Trans. Amer. Soc. of Civil Eng., Vol. 116, pp.770-799, 1951.

18. D.L. Isaacson and R.W. Madsen, Markov Chains: Theory and Applications, John Wiley \& Sons, New York, 1976.

19. ISO/IEC, Information Technology -Coding of moving pictures and associated audio for digital storage media at up to about 1.5 Mbit/s-, ISO/IEC 11172, 1993.

20. ISO/IEC, Information Technology-Generic Coding of Moving Pictures and Associated Audio Information, ISO/IEC 13818, 1994.

21. IVS Baseline Document. Working Party XV/1 Experts Group for ATM Video Coding, CCITT, June 1992.

22. B. Jabbari, F. Yegenoglu, Y. Kuo, S. Zafar, and Y. Q. Zhang, "Statistical Characterization and Block-Based Modeling of Motion-Adaptive Coded Video," IEEE Trans. on Circuits and Systems for Video Tech., vol. 3, no. 3, pp 199-207, June 1993.

23. P.R. Jelenkovic and B. Melamed, "Algorithmic modeling of TES processes," IEEE Trans. on Automated Control, Vol 40, No 7, pp.1305-1312, 1995.

24. F. Kishino, K. Manabe, Y. Hayashi and H. Yasuda, "VBR Coding of Video Signals for ATM Networks," IEEE J. Sel. Areas of Commun., Vol. 7, No. 5, pp 801-806, June 1989.

25. Marwan Krunz, Ron Sass, and Herman Hughes, "Study of VBR MPEG-coded video traffic and associated multiplexing performance," Computer Systems Science and Engineering, Vol. 11, pp. 135-143, May 1995.

26. A. Lazar, G. Pacifini and D. E. Pendarakis, "Modeling video Sources for Real-Time Scheduling," IEEE Globecom, Vol. 2, pp. 835-839, 1993.

27. David M. Lucantoni, Marcel F. Neuts and Amy Reibman, "Methods for Performance Evaluation of VBR Video Traffic Models," IEEE/ACM Transactions on Networking, Vol. 2, No. 2, pp. 176-180, April 1994.

28. Didier Le Gall, "MPEG: A Video Compression Standard for Multimedia Applications," Commun. of the ACM, Vol. 34, No. 4, pp 60-63, April 1991.

29. W.E. Leland, M.S. Taqqu, W. Willinger, and D.V.Wilson, "On the Self-Similar Nature of Ethernet Traffic," SIGCOMM, pp.183-193, 1993.

30. N. Likhanov, B. Tsybakov and N. D. Georganos, "Analysis of an ATM Buffer with Self-Similar ("Fractal”) Input Traffic," IEEE INFOCOM, Vol 3, pp. 985-992, 1995.

31. Ming Liou, "Overview of the px64 kbits/s Video Coding Standard," Commun. of the ACM, Vol. 34, No. 4, pp 60-63, April 1991.

32. B. Maglaris, D. Anastassiou, P. Sen, G. Karlsson and J.D. Robbins, "Performance Models of Statistical Multiplexing in Packet Video Communications," IEEE Trans. on Commun., Vol 36, No 7, pp.834-843, July 1988. 
33. B.B. Mandelbrot and J.W. Van Ness, "Fractional Brownian Motions, Fractional Noises and Applications," SIAM Review, Vol 10, pp. 422-437., 1968.

34. Nasser M. Marafih, Ya-Qin Zhang and Raymond L. Pickholtz, "Modeling and Queueing Analysis of Variable-Bit-Rate Coded Video Sources in ATM Networks," IEEE Transactions on Circuits and Systems for Video Technology, Vol. 4, No. 2, pp. 121-128, April 1994.

35. B. Melamed, "An Overview of TES Processes and Modeling Methodology," Performance Evaluation of Computer and Communications Systems, Springer-Verlag Levture Notes in Computer Science, pp 359-393, 1993.

36. B. Melamed and D. Pendarakis, "A TES-Based Model for compressed Star Wars Video," IEEE GLOBECOM, pp. 120-126, 1994.

37. B. Melamed, D. Raychaudhuri, B. Sengupta, and J. Zdepski, "TES-Based Video Source Modeling for Performance Evaluation of Integrated Networks," IEEE Trans. on Comm., Vol. 42, No. 10, pp. 2773-2777, October 1994.

38. D. G. Morrison, "Variable bit-rate Coding for Asynchronous Transfer Mode Networks," British Telecommun. Tech. J. (UK), Vol. 8, No. 3, pp 70-80, July 1990.

39. Pramod Pancha and Magda El Zarki, "Bandwidth-Allocation Schemes for VariableBit-Rate MPEG Sources in ATM Networks," IEEE Transactions on Circuits and Systems for Video Technology, Vol. 3, No. 3, pp. 190-198, June 1993.

40. J. G. Proakis, Digital Communications, 2nd Edition, McGraw-Hill, 1989.

41. G. Ramamurthy and B. Sengupta, "Modeling and Analysis of a Variable Bit Rate Multiplexer," IEEE INFOCOM, Vol. 2, pp. 817-827, May 1992.

42. D. Reininger, B. Melamed and D. Raychaudhuri, "Variable bit-rate MPEG Video: Characteristics, Modeling and Multiplexing," Proc. of the 14th Intl. Teletraffic Congress - ITC 14, Antibes Juan-les-Pins, France, pp 295-306, 6-10 June 1994.

43. G. Ramamurthy and D. Raychaudhuri, "Performance of Packet Video with Combined Error Recovery and Concealment," In Proc. 6th International Workshop on Packet Video, Portland Oregan, D4.1-D4.3, Sept. 26-27, 1994.

44. Paul Skelly, Mischa Schwartz and Sudhir Dixit, "A Histogram-Based Model for Video Traffic Behavior in an ATM Multiplexer," IEEE/ACM Transactions on Networking, Vol. 1, No. 4, pp. 446-459, August 1993.

45. W. Verbiest, L. Pinno and B. Voeten, "The Impact of the ATM Concept on Video Coding," IEEE J. Select. Areas of Commun., Vol. 6, No. 9, pp. 1623-1632, Dec. 1988.

46. F. Yegenoglu, B. Jabbari, Y. Q. Zhang, "Motion-Classified Autoregressive Modeling of Variable bit-rate Video," IEEE Trans. on Circuits and Systems for Video Tech., vol. 3, no. 1, pp 42-53, February 1993. 